\title{
Los detectives lectores: literacidad crítica y género policial. Actividad didáctica basada en objetos de enseñanza ubicados en la Web (ODE)
}

\author{
Detective Readers: critical literacy and crime \\ fiction genre. Didactic activity based on learning \\ objects located in the Web (ODE)
}

\begin{abstract}
Edith Silveira
Máster en Educación, Universidad ORT Uruguay. Profesora de Literatura, Instituto de Profesores Artigas. Integrante del equipo del Proyecto ProLEE del Consejo Directivo Central de la Administración Nacional de Educación Pública. Miembro del Grupo para la Inclusión y Calidad Educativas a través de Ocuparnos de la Lectura y Escritura en todas las Materias. Integrante de la Red Estrado. Sub directora del Consejo de Educación Secundaria.
\end{abstract}

Fecha de recibido: 12/02/2014

Fecha de aceptación: 11/06/2014

\section{Resumen}

Se trata de la descripción de una propuesta didáctica que se inscribe en la línea de la literacidad crítica como propuesta teórica para la enseñanza de la lectura. Esta línea teórica propone lograr lectores analíticos con una capacidad hermenéutica fluida.

La actividad está pensada para la segunda etapa de enseñanza primaria y se halla presente en la Red.

PALABRAS CLAVE: enseñanza, lectura, literacidad, TIC.

\section{Abstract}

The present work is a description of an educational proposal based on critical literacy as a theoretical proposal for the teaching of Reading skills. It aims at producing analytical readers with fluent hermeneutical skills.

The activity is intended for the second stage of primary education and it can be found on the Net.

KEYWORDS: teaching, reading, literacy, ITC.

\section{Introducción}

He creado la serie de actividades: "Los detectives de la lectura" con la finalidad de potenciar la capacidad de lectura de los alumnos del tercer ciclo de primaria (quinto y sexto año) en el marco de mi trabajo para el programa ProLEE de CODICEN.

La serie se propone realizar una acción potente en cuanto a lograr aprendizajes significativos para andamiar el tránsito a la enseñanza media, por lo que también es una herramienta de equidad. 


\section{¿De qué modo?}

\section{Con un objetivo}

La aplicación de la serie aspira a lograr que la capacidad de lectura de los alumnos consiga desplegar todas las posibilidades del pensamiento tanto en lo que se refiere a la comprensión de un texto como al análisis crítico del mismo.

Las metas de estas actividades seriadas son la autonomía y la apropiación de los distintos tipos de textos por parte de los lectores, es decir la madurez cognitiva de la lectura. Esto se aprecia en la capacidad de realizar inferencias, de distinguir los hechos de las opiniones, de diferenciar la referencia real de la ficción y en la capacidad de llegar a estructurar, de modo autónomo, una interpretación personal fundada en lo leído.

El objetivo educativo en esta concepción es desarrollar la conciencia crítica a partir de la acción de leer un texto.

\section{Con un objeto}

"Detectives de la lectura"1 es una serie de actividades apoyada en el concepto de objeto educativo con característica de blended learning (b-learning), modalidad de enseñanza con soporte informático que combina el modo de educación a distancia con el modo de educación presencial. Para este caso se ha tenido en cuenta la presencia del Plan CEIBAL en las escuelas.

Está realizada con el criterio de la Web 2.0: ODE (Objeto Digital Educativo) y eXeLearning. Ambos recursos son aplicaciones informáticas de uso libre para la enseñanza y permiten crear y practicar actividades en línea.

\section{Descripción del ODE}

Las actividades se desarrollan a partir de lecturas o situaciones de conceptualización gramatical. Son guiadas por dos personajes fijos animados: un zorro llamado "Taimado" y un gorila llamado "Paco". Ellos proponen búsquedas deductivas apoyadas en actividades de lectura sobre el cuento de H. Quiroga "Las medias de los flamencos" y también de conocimiento de la lengua con elementos tomados de la realidad cotidiana. Dentro de la serie existen dos subseries: una dedicada específicamente a las actividades que propician la lectura crítica y otra que desarrolla actividades específicas de conocimiento de la lengua. Se intercala una actividad de lectura y otra de conocimiento de la lengua. El pasaje de una a otra se hace cuando se "gana" una actividad puntual. La dinámica se asemeja -adrede- a la de los juegos de computación.

Las actividades de lectura se realizan con distintas consignas que conducen a determinadas pesquisas, que requieren desde una lectura analítica de textos literarios a trabajos lexemáticos ${ }^{2}$ variados, así como la lectura crítica de textos de la vida cotidiana. Las consignas también conducen a una reflexión sobre la lengua, su uso y sus modalidades.

En este punto es necesario hacer algunas puntualizaciones conceptuales y metodológicas.

\section{Desde la teoría}

\section{Distintos enfoques teóricos acerca de la lectura}

En la actualidad, los últimos avances en lo que se refiere a la lectura aparecen sistematizados en la propuesta realizada por el proyecto OSDE (Open Spaces for Dialogue and Enquiry) consignada en "Critical literacy, Independent thinking, Global citizenship, Global issues and perspectives" (2006). En esta publicación se diferencian tres tipos de lectura a partir de la clasificación de Cervetti, Pardales y Damico (2001):

Lectura tradicional o comprensiva

Lectura crítica

Literacidad crítica (LC) 
La categoría "lectura tradicional", también llamada "lectura comprensiva" (categoría usada generalmente en la escuela para aludir a la práctica de análisis de la comprensión referencial ${ }^{3}$ ) tiene como objetivo desarrollar la comprensión de los contenidos para establecer el valor de verdad en y del texto.

Mi mirada sobre el asunto estima que el nivel de la lectura comprensiva hace ver la lengua como un objeto. Por eso, la lectura en esa concepción se piensa como una operación sobre una materia fija (el texto escrito) y resulta un agente que cumple la función de traducir la realidad.

En el siguiente nivel (la categoría "lectura crítica") lo que se busca es desarrollar la reflexión analítica (la capacidad de percibir las intenciones ${ }^{4}$ y razones que operan en la construcción de un texto determinado) de forma complementaria sin excluir la categoría anterior. Este tipo de lectura tiene en cuenta el contexto, las intenciones y el estilo de la comunicación. Su diferencia con la primera categoría, la de la lectura comprensiva, radica en que avanza hacia una reflexión más profundasobre el lenguaje ${ }^{5}$.

Por último, la categoría "literacidad crítica" (LC de aquí en adelante) suma a estas capacidades enumeradas la de traducir la realidad y la de desarrollar la reflexión analítica, es decir, el conocimiento de que el uso de la lengua es ideológico, entendido el término en su sentido más amplio y que con ella, la lengua, el sujeto y su grupo social construyen una realidad. Por lo tanto, la LC es un tipo de lectura que elabora estrategias de lectura diferentes y es en la construcción de esas estrategias que opera la actividad de "Los detectives de la lectura".

En este tipo de lectura, el significado textual se considera múltiple por el carácter connotativo6 del lenguaje y porque tiene en cuenta el hecho de que el acto de escribir y el de leer se encuentran situados históricamente. La instancia de lectura que se considera en la LC dispara preguntas acerca de la interpretación de un texto y no señala una interpretación en especial como la correcta dentro de un texto.

Para dejar claro este asunto veamos un ejemplo de LC como forma de lectura. El ejemplo está tomado de un texto de Kauffman (inédito 2011) "Leer enseña a pensar". Al describir una posible práctica de lectura en el aula a partir de la fábula de La Fontaine "La cigarra y la hormiga", la citada autora señala que la instancia de lectura comprensiva y las prácticas más comunes de interpretación en este caso conducen a la conclusión de un mensaje de característica moral acerca de las ventajas de la previsión y el trabajo sistemático. Pero esto no excluye -propone Kauffman- que en la categoría LC la instancia de lectura pueda llevar, también, a que un lector crítico se pregunte acerca de la presencia en el texto de una concepción ideológica en relación con el valor del trabajo artístico en la sociedad que lo produce, en un momento histórico dado. (En este caso el trabajo artístico aparece como subestimado).

Esta última interpretación que implica un "leer entrelíneas" es la que la LC propone y aspira a que ayude a evolucionar la capacidad de lectura hasta ese punto en el que una situación que dispara la interrogante conduce a replantear el texto. La LC cuestiona las representaciones mentales trilladas y obvias, los estereotipos, por esto pone al lector en una posición inquisitiva en la que no da nada por sentado y construye una interpretación crítica.

La LC ve la lectura como un medio para apropiarse del mundo y por eso, asimismo, como un medio de transformación social.

En conclusión, la LC es considerada como una práctica social y política que implica el uso de un conjunto de habilidades psicológicas y cognitivas (Casany, 2006; Barton, Hamilton, Ivanič, 2000; Cooper y White, 2008).

El campo de la investigación en la enseñanza de la lectura atiende las tres modalidades indicadas, que no son excluyentes y es en todas estas búsquedas que se sitúa la base teórica de la propuesta didáctica de esta actividad. 


\section{En cuanto a lo metodológico}

En relación con los distintos modos de lectura la situación de un aprendiente de lector no es fácil de determinar. De hecho, en este aspecto la teoría aún vacila sobre un modo de determinar y estimar la situación modal en la operativa de la lectura. Se problematiza el asunto en cuanto a que, en situación, las tres modalidades suelen ocurrir en superposición y de modo alternativo.

En ese sentido el programa ProLEE, en el que se ha creado la actividad, maneja una propuesta de categorización potente. Dentro del trabajo de búsqueda y sistematización del tema, en el programa se han establecido unas pautas llamadas "Referencia de Lectores y Escritores de Español como primera lengua". En dichas pautas la actividad "Los detectives de la lectura" apunta a lectores del nivel 3B7. Las capacidades de lectura de este nivel son las esperadas en el nivel curricular señalado al comienzo; quintos y sextos años de Primaria.

\section{¿Cómo puede un Objeto Digital de Enseñanza aplicado a la lectura ayudar a alcanzar el nivel de la LC?}

Al buscar un soporte para la actividad del Objeto Digital de Enseñanza (ODE de aquí en adelante) me planteé algunas necesidades básicas.

EI ODE necesitaría una estrategia de asedio que fuera comprendida y dominada por los receptores, porque al utilizar la modalidad, b-learning, va de suyo que los participantes necesitan partir de la ZDP (Zona de Desarrollo Próximo) ${ }^{8}$ (Vygotski, 1979), para trabajar con eficacia.

Tracé, de acuerdo a esto, un desarrollo que se apoya en las estrategias de lectura propias del subgénero literario relato policial ya que el relato policial y sus estrategias de recepción trascienden el marco de lo literario pues este tipo de relato está presente también en el cine y particularmente en abundantes series televisivas por lo que podía dar por descontado que se trataba de un asunto conocido para los receptores del ODE y que permitiría el despliegue de acciones propias del b-learning.

Consideré, asimismo, que la relación de la LC con la intriga policial resultaría interesante para lograr estimular la participación y con ella las estrategias que ayuden a alcanzar dicho nivel lector.

Para comprender este asunto es necesario establecer cuáles son las características del subgénero narrativo categorizado como relato policial.

En la narración policial la lectura exige aceptar ciertas condiciones determinadas para el pacto narrativo. En esta lectura lo importante es el desarrollo y no la conclusión final, que es siempre igual y conocida de antemano. La culminación del relato es siempre la identificación y el castigo del mal y el restablecimiento del orden sobre el caos. Este tipo de producción discursiva tiene amplia aceptación en la recepción y por lo tanto existen en los receptores estrategias de lectura y pautas de recepción ya instaladas que se manejan de un modo internalizado. A partir de estas consideraciones determiné que estas formas serían apropiadas para mis intenciones en cuanto a la aplicación del ODE, pues apelando a ese tipo de pacto narrativo tan peculiar existirían ventajas para acceder a las condiciones de lector exigidas por el nivel LC. De este recorte surgen el nombre y las estrategias del ODE: "detectives" preguntando y preguntándose por secuencias y datos relevantes del texto como si fueran pistas que develan el sentido.

Cabe precisar algunos detalles. El personaje del detective (categoría de construcción del relato policial) es reconocible para los receptores, y ocurre otro tanto con sus procedimientos. Tiene una carga positiva de significación: es un héroe literario actual y tiene prestigio como operador deductivo, y en el caso de los productos televisivos más actuales también lo tiene como operador científico. Por estas razones, en nuestro contexto social el relato policial es una serie cultural $^{9}$, lo que posibilita una interactuación con reglas implícitas. 
No es menor el asunto de apoyar el desarrollo de la LC en situaciones de decodificación compartidas por todos los posibles copartícipes en la serie de actividades: estudiantes, maestros y otros adultos que se incorporen a la misma y se interesen como agentes de enseñanza.

En otro aspecto me interesaba lograr que la tecnología se volviera mediadora y gestora de la lectura en lugar de sustituirla.

\section{Desde la didáctica}

\section{La didáctica en general}

La serie de actividades se apoya en los trabajos de Vygotski (1979) y su concepción, ya mencionada, de que los conocimientos se despliegan en relación con las nociones ya presentes (Zona de Desarrollo Próximo) y de que la adquisición del lenguaje es un hecho social y socializante y, por ende, siempre está impregnada por el contexto. Asimismo, Bruner (1988, 1997), a través de la metáfora del "andamiaje" focaliza el proceso de la enseñanza andamiada en dos aspectos: el carácter "necesario" de la ayuda y el carácter "transitorio" de ésta.

Este concepto de "andamiaje" describe las intervenciones tutoriales asimétricas entre maestros y estudiantes en el proceso constructivo de lograr autonomía. De acuerdo con esto, los objetos pedagógicos que integran el conjunto de la actividad "Los detectives de la lectura" son un andamiaje para construir modos de lectura que lleven al nivel autónomo de la LC.

\section{Desde la didáctica de la lectura y la literatura}

Comparto con Mendoza Fillola (2001) que la didáctica de la literatura comienza a partir de la didáctica de la lectura y que hoy existe una relación entre los conocimientos acerca de los procedimientos de lectura señalados por el cognotivismo ${ }^{10}$ y el enfoque teórico de la Teoría de la recepción formulada por Jauss e Iser (1987). Según estos autores, la conjunción de ambos trazados permite plantear una didáctica diferente a la filológica para la enseñanza de la literatura en la institución escolar.

Estos aportes se reflejan en la categorización que plantea Mendoza Fillola (2001) de competencia literaria e intertexto lector.

La educación literaria hay que entenderla como un proceso que incluye distintas facetas, pero que siempre está muy en dependencia respecto a la formación del lector. La atención didáctica de la formación del lector se ha centrado en la actividad del lector y en los componentes que intervienen en los procesos de recepción. Y, puesto que los criterios de valoración estética están en dependencia de los convencionalismos estéticos culturales y de criterios artísticos, resulta que la intervención didáctica correspondiente a la educación literaria necesariamente se halla relacionada con la funcionalidad (activación y desarrollo) del intertexto lector.

Cabe señalar que la categoría de "competencia literaria" implica, según la concepción de este autor, una combinación de elementos y sucesos que involucran una actividad personal que se produce a partir de la puesta en práctica de determinados conocimientos. Éstos responden a asociaciones contextuales y culturales a partir de lo mencionado en el texto. La búsqueda de significados siempre parte de establecer relaciones que se pueden dar por oposición o por contigüidad dentro del texto. Estas relaciones deben ser detectadas en la acción de reconstrucción que representa el hecho de leer.

El concepto de "intertexto" refiere a la concepción de Genette (1982) y de Rifaterre (1990) (Ápud Mendoza Fillola, 2001) "el intertexto se definió como el conjunto de textos que entran en relación con el texto dado (...) el fenómeno literario, en todos los casos es una dialéctica entre el texto y el lector". 


\section{Conclusiones}

Por lo expuesto, la serie de actividades propuesta tiene como objetivo la mejora y ampliación de las prácticas de lectura en el ámbito de Enseñanza Primaria, para lograr que los estudiantes puedan desplegar todas sus capacidades de análisis y pensamiento a través de estrategias más amplias y personales de lectura y que lleguen a la Enseñanza Media con mejores herramientas y con macrohabilidades que les permitan establecer andamiajes para las nuevas demandas de los aprendizajes.

También aspira a la búsqueda de nuevos modos de mediar y andamiar conocimientos para todos los estudiantes como un modo de potenciar las transformaciones cognitivas necesarias para el crecimiento de los proyectos personales.

Mi búsqueda continúa en este camino hoy desde un proyecto de enseñanza de la lectura en un liceo macro de Montevideo, con la consigna del cuerpo docente para una meta posible de "Mejorar la lectura de todos los signos para toda episteme".

Espero que esta intervención sea un disparador para la reflexión y la investigación en un campo de la enseñanza que es un real desafío pedagógico en nuestra realidad.

\section{Bibliografía}

Allison, D. (2008). ESL students and critical literacy: a case study of critical writing. (Thesis _PhD/Research) Unpublished.

Barton, D., Hamilton, M. \& Ivanič, R. (2000). (EDS) Situated Literacies: Reading and Writing in Context. Londres. Routledge.

Bordieu, P. (1988) La distinción. Criterios y bases sociales del gusto. Madrid. Taurus.

Bruner, J. (1988). Desarrollo cognitivo y educación. Madrid. Ediciones Morata.

Bruner, J. (1997). La educación puerta de la cultura. Madrid. Visor.

Cassany, D. (2006). Tras las líneas. Barcelona. Anagrama.

Cervetti, G., Pardales, M.J. y Damico, J.S. (2001)A tale of differences: Comparing the tradition, perspectives, and educational goals of critical reading and critical literacy. Disponible en: http:// www.readingonline.org/articles/art_index.asp?HREF=/articles/cervetti/index.html

Consultado el 25/5/2014

Cooper, K. \& White, R. (2008). Critical Literacy for school improvement: an action research Project. En: Improving Schools, SAGE Publications. 11(2), pp 101-113.

Genette, G. (1971). Figures III. Paris. Éditions du Seuil.

Iser, W. (1987). El acto de leer. Madrid. Taurus.

Jakobson, R. (1975). Ensayos de lingüística general. Barcelona. Seix Barral.

Jauss, H. R. (2000). La historia de la literatura como provocación. Barcelona. Ediciones Península.

Kauffman, R. (2001). Leer enseña a pensar. Inédito. Documentos compartidos producidos por el grupo de investigación en ProLEE. Montevideo. 
Mendoza Fillola, A. (2001) El intertexto lector. M.E. García Gutiérrez (Coord.) La educación lingüística y literaria en secundaria: materiales para la formación del profesorado. Vol. II. La educación literaria. Disponible en: file://C:/Users/EDIT/Downloads/396-Texto\%20Completo\%20 1\%20La\%20educación\%20lingüística \%20y\%20literaria \%20en \%20secundaria \%20-\%20 materiales\%20para\%20la\%20formación Consultado: 25/05/2014.

Osde Project (2006). Critical literacy, Independent thinking, Global citizenship, Global issues and perspectives. Professional Development Resources Pack. Disponible en: http://www. osdemethodology.org.uk/ Consultado 25/05/2014.

Vygotski, L. S. (1973). Pensamiento y lenguaje. Buenos Aires. La Pléyade.

Vygotski, L. S. (1979). El desarrollo de los procesos psicológicos superiores. Barcelona. Crítica.

\section{Notas}

${ }^{1}$ Se puede acceder a la serie en el siguiente link: http://www.uruguayeduca.edu.uy/Portal.Base/ Web/verContenido.aspx?ID=211144

${ }^{2}$ Lexemático, sobre cada palabra en sí misma.

${ }^{3}$ En el sentido de lo que Jakobson señala como función referencial del lenguaje. La función referencial del lenguaje consiste en representar o manifestar objetivamente la realidad exponiendo hechos y conceptos.

${ }^{4}$ Intención, en este caso se refiere a lo que se propone lograr en la recepción, es decir el efecto en el lector y no al propósito del productor del texto.

${ }^{5}$ Reflexión metalingüística en términos técnicos.

${ }^{6}$ Connotar 1) tr. ling. Sugerir una palabra, frase o discurso un significado añadido y distinto al suyo propio, literal y estricto. Ej. "Sus palabras connotan buenas intenciones". El diccionario utilizado es Word Reference, http://www.wordreference.com.

${ }^{7}$ Para aclarar la tipificación ver: http://www.uruguayeduca.edu.uy/repositorio/prolee/pdf/ pautas_\%20de_referencia.pdf

${ }^{8}$ Zona de desarrollo próximo

${ }^{9}$ En el sentido que lo menciona Bordieu.

${ }^{10}$ Este enfoque se plantea preguntas que aspiran a lograr una reflexión cognitiva por parte del lector. Se solicita al lector identificar la intención del autor (en cuanto a la finalidad de la comunicación, es decir, identificar los procedimientos incluidos en el texto, persuasión, argumentación, información, etc.) relacionar elementos textuales o temáticos, pensar, reflexionar, hipotetizar casos, hechos y fenómenos, interpretar y expresar acuerdo, relacionar causa y efecto, etc. Se le asigna una alta importancia a la metacognición como un proceso que permite controlar y regular la comprensión, ya que el objetivo principal de la lectura, para esta posición, es la comprensión del texto. 